\title{
Spectroscopic properties of thin glass films doped by laser dyes prepared by sol-gel method
}

\author{
R. REISFELD ${ }^{(1)}$, D. SHAMRAKOV and Y. SOREK \\ Department of Inorganic Chemistry, The Hebrew University of Jerusalem, 91904, Israel
}

As presented at the second French-Israeli workshop on Solid State Lasers, glass waveguides can be prepared at low temperature. Here we present optical properties of laser dyes - perylimide dye lumogen LFR300, xantene dye Rhodamine $6 \mathrm{G}$ and merocyanine dye DCM - incorporated into glass waveguides. Examples of superradiance, amplification and laser emission in the films are demonstrated.

Introduction

In a foregoing paper [1] we have outlined the numerous applications of thin glass films doped by laser dyes. We have also emphasized the advantage of glass wave guides over a classical hosts mainly liquid and polymeric matrices. The relevant references are included in [1-3]. The present paper gives several concrete examples of laser dyes: perylimide, xantene and merocyanine, incorporated into wave guides with the refractive index higher than that of the support. The synthesis of the films was performed by copolymerisation of titanium alkoxydes with organically modified silanes (ormosils) [2]. Such a system after hydrolisis and polycondensation forms glass films at room temperature allowing incorporation of the appropriate dyes during the process of glass formation.

Another wave guiding structure can be prepared by deposing glass films based on silica - polymethylmethacryilate composite [3] on quartz plates having lower refractive index than the film into which the dyes were introduced on the stage of coating solution formation.

\section{Experimental}

Wave guiding films were prepared either by copolymerysation of the ormosil - $\gamma$ GLYcidoxypropyltriMethOxysilane (GLYMO) with titanium alkoxide [2] or by combining the organically modified silica with the Polymethylmethacrylate [3]. The support glasses or quartz plates were covered by the films by dip or spread coating. The composition of coatings can be varied widely providing the refractive index control and thus desirable wave guiding properties. The coating solutions contained a variety of dyes including perylimide dyes (lumogen LFR 300 and LFO 240 (BASF)), Rhodamines (Rhodamine 6G, Rhodamine 610), DCM, Kiton red and acridines.

(1) Enrique Berman Professor of Solar Energy 
Optical measurements of amplification, lasing threshold energy, spectral narrowing and tunability were performed using the frequency doubled Nd:YAG laser (Lumonix HY 600), Si PIN photodetector, Tektronix TDS 620 digitizing oscilloscope and grating monochromators.

A significant spectral narrowing of the guided ray that either evolves from the film edge or from a prism coupler was observed in cases when the threshold energy was reached. Two examples are presented in Fig. 1,2 for the LFO 240 dye in the composite film and DCM dye in the titania - GLYMO ormocer film correspondingly.

The waveguiding laser output can be tuned using the distributed feedback (DFB) technique [4] by gain modulation along the excited zone satisfying the resonant backward Bragg scattering conditionaccording to equation:

$$
\lambda=2 \mathrm{n} \Lambda \text {, (1) }
$$

(where $\Lambda$ is the period of the spatial modulation of the gain ). Thus the emission frequency of the DFB laser can be tuned by altering the period $\Lambda$.

The necessery gain modulation within the active wave guide can be induced when it is pumped with interference fringes formed by augmented crossing of previously split plane coherent pumping beam

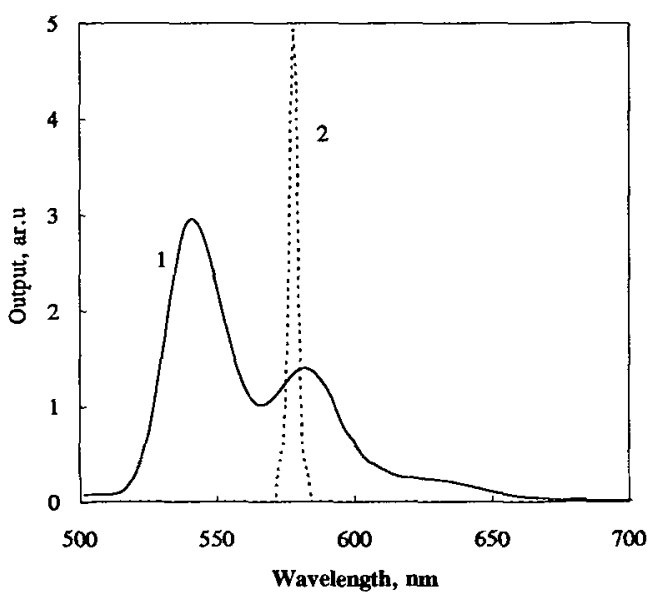

Fig. 1. Spontaneous (1) and Stimulated (2) emission spectra of LFO240 dye in polymer - silica composite deposited on quartz.

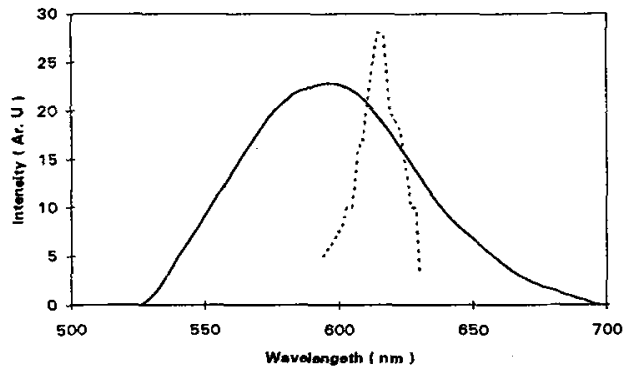

Fig. 2. Spontaneous fluorescence of DCM: (solid line ) and the guided ray spectrum (dashed line). at the angle:

$$
\theta=\arcsin \left(\lambda_{\mathrm{p}} / 2 \Lambda\right)
$$

where $\lambda_{p}$ is the wavelength of the pump radiation. It was done with aid of quartz prism attached to the uncoated plate face as shown in Fig. 3. The tunability in the range of 615 $629 \mathrm{~nm}$ was observed for the LFR 300 dye in the composite glass film coated on quartz plate. The tuning was obtained at incident angles $\mathbf{i}=15-25^{\circ}$ of external pumping. The tunability was achieved so far at rather narrow spectral range close to the gain maximum of the dye applied. The tuning range of $14 \mathrm{~nm}$ obtained here could be obviously broadened by optical configuration optimizing. 
The relative output energy dependence on the pumping energy was measured for various dyes in titania - GLYMO wave guides. A typical example of threshold behavior is shown in Fig. 4 for Rhodamine 610. The threshold energy values ranged between 30 to 60 $\mu \mathrm{J} /$ pulse for dye concentrations of about $10^{-3} \mathrm{M}$ in the coating solutions .

Amplification of guided He-Ne laser beam was studied in LFR 300 doped ormocer film as described in [2]. Despite the fact that the overlap between the LFR 300 fluorescence and the He-Ne laser wavelengths is rather poor a significant amplification up to $60 \%$ was observed under about $2 \mathrm{~mJ} /$ pulse pumping. The amplified stimulated emission was coherent.

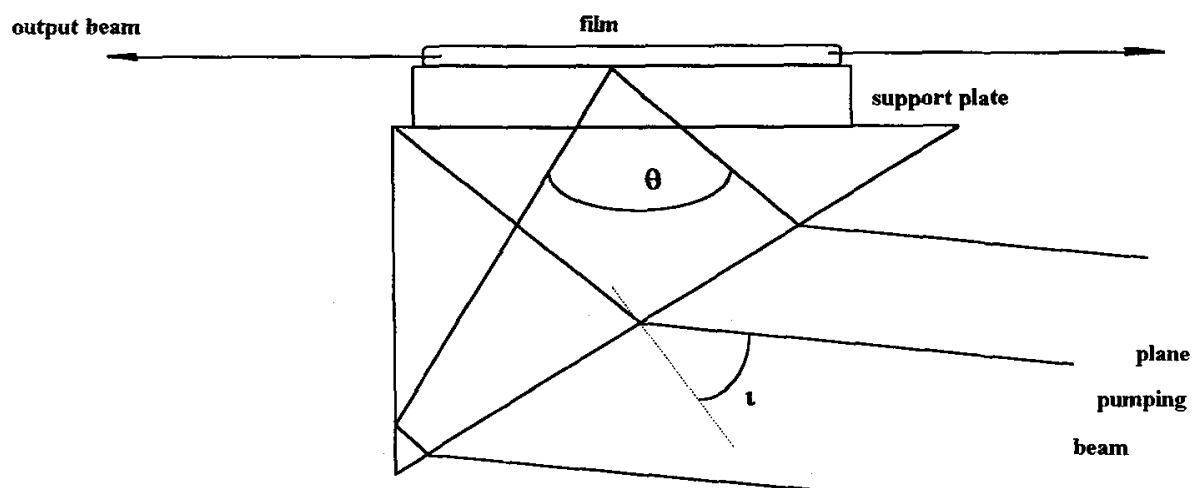

Fig. 3 Scheme of tunable DFB Laser device. Active composite glass wave guide (the profile enlarged) is attached to the quartz prism by its support. $\theta$ - crossing angle of two coherent beams producing the exciting interference pattern within the film. $\mathbf{i}$ - external pumping incidence.

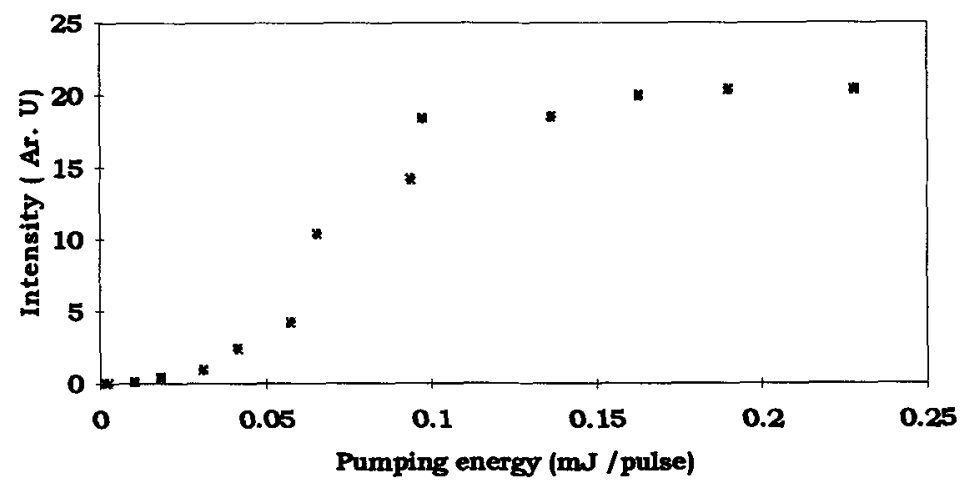

Fig.4. The relative output energy versus the pumping energy for Rhodamine 610 in ormocer film. 
Conclusions

We present here a novel way for preparation of active wave guides and demonstarte their performance with a number of laser dyes. It should be noted that a rapid development of new powerful diode lasers especially emitting in the blue part of the spectrum, opens a possibility for compact wave guide laser devices based on the results reported here. It means the exciting source can be an integral part of the device.

\section{Acknowledgments}

The work was supported by the US Army European Research Office and Night Vision Laboratory contract DAJA 45-90-C-055 and by Israeli Ministry of Absorption.

We want also to thank Mrs. I.Finkelstein for her assistance in theamplification measurements.

\section{References}

[1] R.Reisfeld, Wave Guided Sol-Gel Glass Lasers. Paper presented at M2P Lyon 1993, to be published in this volume.

[2] Y.Sorek, R.Reisfeld et al. Active Glass Waveguides Prepared By the Sol-Gel Method.Proc. II Binational French-Israeli Workshop on Solid State Lasers. Lyon Dec. 5-8 1993.

[3] D.Shamrakov and R.Reisfeld, Super Radiant Film Laser Operation of Perylimide Dyes Doped Silica-Polymethylmethacrylate Composite. Proc. II Binational French-Israeli Workshop on Solid State Lasers. Lyon Dec. 5-8 1993.

[4] H.Kogelnik, C.V.Shank, Stimulated emission in a periodic structures, Appl. Phys. Lett., 18(1971), N4, 152. 